Service social

Psychologie gérontologique, par Jean Vézina, Philippe

Cappeliez et Philippe Landreville, Boucherville, Gaëtan Morin éditeur, 1994, 459 pages.

\title{
Daniel Pelletier
}

Volume 43, numéro 1, 1994

Maintien à domicile

URI : https://id.erudit.org/iderudit/706647ar

DOI : https://doi.org/10.7202/706647ar

Aller au sommaire du numéro

Éditeur(s)

École de service social de l'Université Laval

ISSN

1708-1734 (numérique)

Découvrir la revue

Citer ce compte rendu

Pelletier, D. (1994). Compte rendu de [Psychologie gérontologique, par Jean

Vézina, Philippe Cappeliez et Philippe Landreville, Boucherville, Gaëtan Morin éditeur, 1994, 459 pages.] Service social, 43(1), 143-145.

https://doi.org/10.7202/706647ar d'utilisation que vous pouvez consulter en ligne.

https://apropos.erudit.org/fr/usagers/politique-dutilisation/ 


\section{RECENSIONS}

\section{PSYCHOLOGIE GÉRONTOLOGIQUE}

Jean Vézina, Philippe Cappeliez et Philippe Landreville Boucherville, Gaëtan Morin éditeur, 1994, 459 pages.

La gérontologie et l'étude de la psychologie des personnes âgées constituent des sujets de plus en plus présents dans les préoccupations des intervenants ${ }^{1}$. De nos jours, il reste cependant difficile de trouver des ouvrages qui rassemblent les connaissances actuelles sur les thématiques du vieillissement, de la psychologie et des personnes âgées, dans une perspective qui soit à la fois scientifique et critique, sans se tourner vers une littérature et des revues scientifiques spécialisées.

La parution récente de l'ouvrage de Jean Vézina, Philippe Cappeliez et Philippe Landreville (1994) comble ce vide et apporte une réponse aux étudiants et intervenants qui s'interrogent sur les différents concepts et théories d'une psychologie gérontologique en pleine évolution. Ces trois auteurs québécois réalisent ainsi une première en rassemblant dans un même ouvrage les connaissances et théories de base de cette jeune science. Ils nous guident vers une meilleure compréhension des dimensions théoriques et pratiques que ces questions soulèvent dans la littérature scientifique. L'ouvrage se distingue des quelques ouvrages de psychologie du vieillissement actuellement disponibles en adoptant une perspective résolument scientifique et axée sur l'analyse des concepts et des données de recherches récentes en psychologie.

Cet ouvrage des plus formateurs offre une intéressante synthèse des connaissances actuelles sur une douzaine de thèmes choisis en psychologie gérontologique. De niveau universitaire, tant par la critique des recherches en psychologie que par la qualité de la documentation et la rigueur de la démarche, cet ouvrage réalise une analyse étoffée

1. L'utilisation du masculin des termes a été privilégiée dans ce texte pour en simplifier la lecture. Cette dénomination inclut alors le masculin et le féminin du terme. 
de thèmes pertinents à ce domaine. Les auteurs explorent ainsi les aspects démographiques et les attitudes envers les personnes âgées, les processus sensoriels, la sexualité, I'intelligence, la mémoire, la personnalité, le bien-être, les transitions que peut vivre une personne âgée - dont la mort du conjoint, la retraite, le relogement et la maladie physique -, la solitude, la dépression, le suicide et les pathologies cérébrales organiques telles les démences et la maladie d'Alzheimer. Ils passent en revue les différentes notions de base, posent les enjeux méthodologiques et remettent en question concepts et recherches pour mieux dégager les facteurs qui affectent le fonctionnement psychologique, social, intellectuel et affectif de la personne âgée. Ils mettent en évidence les modifications apportées par l'âge, tout en rappelant les limites des méthodes et des concepts utilisés par les chercheurs.

Cette analyse se situe parfois aux frontières de la neuropsychologie en abordant les phénomènes des pertes sensorielles, de la perception, de la mémoire et de la personnalité comme autant de composantes de l'adaptation et du bien-être de la personne âgée. Cet ouvrage présente ici un intérêt certain pour l'étudiant, le chercheur ou l'intervenant qui veut prendre connaissance de ces sujets, passer en revue les théories et notions pertinentes. Le livre Psychologie gérontologique constitue alors une source importante d'informations et de références récentes.

La structure thématique des chapitres accompagne aisément une démarche pédagogique pour un cours de psychologie du vieillissement. Chacun des chapitres offre un court sommaire de son contenu et est complété d'un résumé, de suggestions de lectures et d'une abondante bibliographie. Enrichi de tableaux et schémas, cet ouvrage comprend des synthèses judicieuses des recherches actuelles et illustre les modèles et concepts liés à chacun de ces sujets.

Il est à noter qu'il demande au lecteur une certaine familiarité avec des concepts de base utilisés en psychologie, de même qu'une lecture attentive. Afin que chacun saisisse rapidement les différentes dimensions de chacun des thèmes, les auteurs ont cru bon de présenter les fondements conceptuels des thèmes et de signaler, en caractères gras, les notions centrales. On y trouve alors des exposés d'une grande clarté qui permettent de saisir les différents concepts et théories qui alimentent la recherche actuelle.

Pour avoir enseigné au cours des quinze dernières années la psychologie et la gérontologie, tant au niveau collégial qu'universitaire, je considère Psychologie gérontologique comme un ouvrage à la fois dense et d'une grande clarté pour aborder les sujets pertinents de ce domaine en constante évolution. Les intervenants auprès des personnes âgées y trouveront une source précieuse $d^{\prime}$ information pour mieux comprendre le fonctionnement de la personne âgée dans ses 
fondements psychologiques et voir l'apport de la recherche dans ce domaine. Je suis sûr que cette lecture suscitera de nouvelles interrogations et un plus grand intérêt pour les aspects particuliers du fonctionnement cognitif, normal ou pathologique de la personne âgée.

Daniel PELLETIER

Centre de recherche sur les services communautaires

Université Laval

\section{LES PERSONNES ÂGÉES. DÉPENDANCE, SOINS ET SOLIDARITÉS FAMILIALES. COMPARAISONS INTERNATIONALES}

Sous la direction de Frédéric Lesemann et Claude Martin

Paris, Les études de La Documentation française, 1993, 215 pages.

Le vieillissement des populations renforce aujourd'hui la crise des Étatsprovidence et impose une réflexion sur les systèmes de protection sociale. Dans le domaine de la prise en charge des personnes âgées dépendantes, cette évolution conduit à la redécouverte et à la reconnaissance du rôle des familles par les pouvoirs publics.

À une logique mécanique de substitution, cet ouvrage oppose une logique de complémentarité entre solidarités familiales et solidarités publiques. En cela, la dynamique des contributions familiales se révèle spécifique et non réductible à celle des services.

Voilà esquissée, au dos de cet ouvrage collectif, la perspective retenue. Le livre porte sur un bilan international de recherches ayant pour objet l'implication des familles à l'endroit des personnes âgées en perte d'autonomie, tout particulièrement dans le contexte des rapports qui existent entre l'État et les familles, dans huit pays industrialisés. Tour à tour, les expériences de la France, de l'Allemagne, du Royaume-Uni, de I'Italie, de la Suède, des Pays-Bas, du Canada et des États-Unis sont présentées pour rendre compte à la fois de la façon dont les systèmes nationaux tentent de répondre aux besoins de prise en charge des personnes âgées dépendantes et de la manière dont les familles participent à cette prise en charge. La diversité des expériences recensées a tôt fait d'illustrer que la culture de ces derniers pays modèle le rapport État-familles dans leurs politiques sociales; paradoxalement, des constantes, des évolutions analogues et des interrogations communes s'imposent tout au long du parcours.

Qui « doit» s'occuper des personnes âgées en perte d'autonomie? Qui s'en occupe de fait? Dans quelles conditions et à quel prix? Quelle 